EUROPHYSICS LETTERS

00002001

Europhys. Lett., 00 (0), pp. 000-000 (2000)

\title{
Counterions at charge-modulated substrates
}

\author{
André G. Moreira ${ }^{1,2}$ And Roland R. Netz ${ }^{1,3}$ \\ ${ }^{1}$ Max-Planck-Institut für Kolloid- und Grenzflächenforschung, 14424 Potsdam, Germany \\ ${ }^{2}$ Materials Research Laboratory, UCSB, Santa Barbara, CA 93106, USA \\ ${ }^{3}$ Sektion Physik, LMU, Theresienstr. 37, 80333 München, Germany
}

(received 0000 2001; accepted )

PACS. 82.70.-y - Disperse systems; complex fluids.

PACS. 61.20.Qg - Structure of associated liquids: electrolytes, molten salts, etc..

PACS. 82.45.+z - Electrochemistry.

\begin{abstract}
We consider counterions in the presence of a single planar surface with a spatially inhomogeneous charge distribution using Monte-Carlo simulations and strong-coupling theory. For high surface charges, multivalent counterions, or pronounced substrate charge modulation the counterions are laterally correlated with the surface charges and their density profile deviates strongly from the limit of a smeared-out substrate charge distribution, in particular exhibiting a much increased laterally averaged density at the surface.
\end{abstract}

The recently revived interest in charged soft-condensed matter systems reflects that there are still many open questions, despite the enormous amount of work available in this field. One example of high experimental relevance is the discrete nature of charged surface groups, or, more generally, the inhomogeneity of substrate charge distributions, and how it affects various thermodynamic properties in an aqueous environment such as forces between charged particles or the counterion distribution. The importance of the discreteness of charged surface groups has been experimentally established in colloidal flocculation[1] and deposition studies[2] and was recently reviewed[3]. On a much larger length scale, chemically micropatterned substrates with charged and neutral patches can be used for controlled colloidal deposition[4] and DNA immobilization[5]. Theoretically, charge-modulated surfaces have been studied by various mean-field approximations $[6,7,8,9,10]$, liquid state theory[11, 12], as well as computer simulations $[13,14,15]$. In these studies, the importance of inhomogeneous surface charge distributions has been recognized, and for a number of different charge-distribution models and parameters the ionic distribution functions as well as forces between charged surfaces have been calculated. In this paper we use a simple model for a charge-modulated surface which depends on a single geometric parameter and includes as limiting cases both smeared-out and deltapeaked charge distributions. We study the distribution of counterions without added salt at a single charged surface for all different values of the electrostatic coupling parameter (depending on temperature and counterion valence) and for different degrees of surface charge modulation, both using Monte-Carlo (MC) simulation methods and the recently introduced strong-coupling 
(SC) theory $[16,17,18]$. Our study therefore encompasses different experimental situations such as mono/multivalent ions at surfaces with discrete charged chemical groups, as well as charged colloids at microscopically charge-modulated surfaces.

As we will demonstrate, the assumption of smeared-out charges dramatically breaks down when the charge-modulation at the surface is pronounced, but also for moderate substratecharge modulation when the electrostatic coupling is large (i.e. for highly charged surfaces, low temperatures, or multivalent counterions): in both cases the counterions become highly correlated with the surface charges and tend to form a two-dimensional, laterally ordered layer close to the surface. A convenient measure for the effects of substrate charge modulation is the counterion contact density, i.e., the laterally averaged counter-ion density at the substrate surface, since it is known exactly in the smeared-out case and can be easily determined from simulations[16]. We find that the contact density for charge-modulated substrates can be much larger than for the smeared-out case, which is in agreement with recent experimental measurements on highly charged surfactant monolayers[19]. Quite surprisingly, substrate charge modulation tends to have a more drastic effect on the counter-ion distribution than fluctuations and correlations, which have been the subject of numerous recent studies (see [16] and references therein). As our numerical results show, the SC theory describes the counterion distributions quantitatively in the SC limit and in particular in the limit of pronounced surfacecharge modulation. We also consider a dielectric-constant jump at the substrate, as relevant for charged biological and colloidal surfaces[20, 21, 22, 23]. In contrast to the mean-field Poisson-Boltzmann (PB) approach, which severely fails even in the smeared-out case, our SC approach compares well with numerical results and helps to understand the intricate interplay of dielectric-jump and charge-modulation effects.

The substrate charge distribution is modeled, for simplicity, by point charges distributed on a square lattice with lattice constant $a$. The point-like oppositely charged counterions are confined to the positive half-space with $z>0$, the minimal distance between surface charges and counterions is given by $D$, as shown in Fig. 1a. The dielectric constant of the positive half-space $\varepsilon_{>}$is allowed to be different from $\varepsilon_{<}$. The system is globally neutral, and only the fixed surface charges and counterions are present, i.e., no salt is added. In principle, one could interpret $D$ as the sum of the surface-ion and counterion hard-core radii. In our model, however, we use the ratio $D / a$ more generally to control the degree of substrate-charge modulation, which allows to describe a variety of experimental situations and systems by a single parameter. The limit $D / a \rightarrow \infty$ is equivalent to a smeared-out surface-charge distribution, while $D / a \rightarrow 0$ corresponds to a delta-peaked distribution.

The typical height of the counterion layer in the smeared-out limit defines the GouyChapman length $\mu=1 /\left(2 \pi q \ell_{B} \sigma_{s}\right)$, where $\sigma_{s}=Q / a^{2}$ is the number charge density at the wall, $q$ and $Q$ are the valences of counterions and surface ions, and $\ell_{B}=e^{2} / 4 \pi \varepsilon_{>} \varepsilon_{0} k_{B} T$ is the Bjerrum length (the distance at which two elementary charges interact with thermal energy, $\left.k_{B} T\right)$. In the following, we will rescale all lengths by $\mu$ according to $\widetilde{r} \equiv r / \mu$. For the simple double layer (smeared out charges at the surface and no dielectric jump) the coupling parameter $\Xi=q^{2} \ell_{B} / \mu=2 \pi q^{3} \ell_{B}^{2} \sigma_{s}$ is the only parameter in the problem. In the limit $\Xi \rightarrow 0$ (weakly charged surfaces, low-valence counterions, or high temperatures) the PB theory is asymptotically exact, while in the opposite limit $\Xi \rightarrow \infty$ (highly charged surfaces, high-valence counterions, or low temperatures) the SC theory is exact[16]. The present model is in addition characterized by $D / a$, the dielectric-constant ratio $\Delta=\left(\varepsilon_{>}-\varepsilon_{<}\right) /\left(\varepsilon_{>}+\varepsilon_{<}\right)$and the valence ratio $q / Q$ between counterions and surface charges.

The Coulomb interaction in the presence of dielectric discontinuities is given by the solution of the Poisson equation with the appropriate boundary conditions[24]. For a single dielectric jump located at $z=0$ as shown in Fig.1a, the electrostatic energy reads 

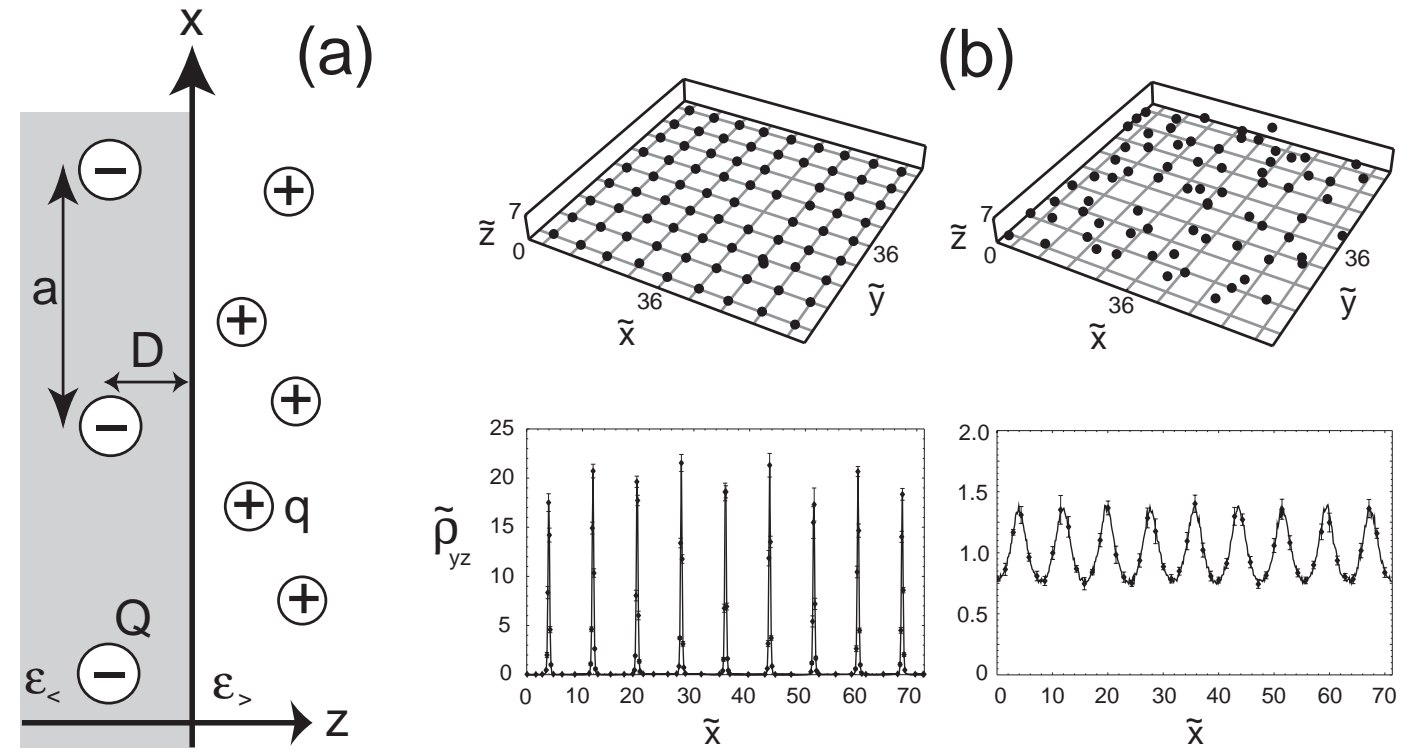

Fig. 1. - a) In our model a square lattice of fixed surface charges with lattice constant $a$ is located in the $x y$-plane at a distance $D$ from the counterion containing half space. b) Simulation snapshots and normalized lateral density profiles $\widetilde{\rho}_{y z}(\widetilde{x})$ (averaged in the $y z$-plane) for a coupling constant $\Xi=10$, vanishing dielectric jump $(\Delta=0)$ and valence symmetry between surface and counterions $(Q=q)$ for $D / a=0.06$ (left) and $D / a=0.24$ (right), showing strong and weak lateral counterion ordering, respectively. In the snapshots, the surface ions are located beneath the lattice nodes.

$$
\frac{\mathcal{H}}{k_{B} T}=\Xi \sum_{i>j}\left\{\frac{1}{\left|\widetilde{\mathbf{r}}_{i}-\widetilde{\mathbf{r}}_{j}\right|}+\frac{\Delta}{\sqrt{\left[\widetilde{\mathbf{r}}_{i}-\widetilde{\mathbf{r}}_{j}\right]^{2}+4 \widetilde{z}_{i} \widetilde{z}_{j}}}\right\}-\Xi(1+\Delta) \frac{Q}{q} \sum_{i, \alpha} \frac{1}{\left|\widetilde{\mathbf{r}}_{i}-\widetilde{\mathbf{R}}_{\alpha}\right|}+\sum_{i=1}^{N} \frac{\Xi \Delta}{4 \widetilde{z}_{i}}
$$

The first sum corresponds to the interaction between pairs of counterions at positions $\mathbf{r}_{i}$ (taking into account the so-called "image charges" through the term proportional to $\Delta$ ), the second sum is the interaction between counterions and fixed surface charges (the sum over $\alpha$ corresponds to all surface-ion lattice vectors $\widetilde{\mathbf{R}}_{\alpha}$ ) and the third sum is the interaction between counterions and their "images." In our MC simulations we use Eq. (1) for a typical number of 100 counterions in conjunction with periodic boundary conditions (implemented by the Lekner-Sperb technique[25]) in order to minimize finite size effects[16]; the results reported here are always taken from runs with $10^{6}$ Monte Carlo steps per particle. In Fig. $1 b$ we show the effects of varying surface-charge modulation: For a ratio $D / a=0.24$ (to the right) the counterion snapshot shows a rather irregular configuration, with no or little visible correlation between surface charges (located beneath the nodes of the square lattice) and counter ions. For a ratio $D / a=0.06$ (to the left), on the other hand, the counterions are strongly correlated with the square lattice of the surface ions (two counterions obviously have, driven by thermal fluctuations, escaped from their assigned lattice positions). This is reflected by the normalized lateral counterion distribution $\widetilde{\rho}_{y z}(\widetilde{x})$, which for $D / a=0.24$ oscillates weakly around its mean value of unity, while for $D / a=0.06$ this distribution function is strongly peaked at the positions of the surface ions.

Before we present the density profiles as a function of the distance from the substrate, we quickly recapitulate the SC theory. At leading order of an expansion in inverse powers of the coupling strength $\Xi$, which is equivalent to a virial expansion, the normalized counterion 
EUROPHYSICS LETTERS
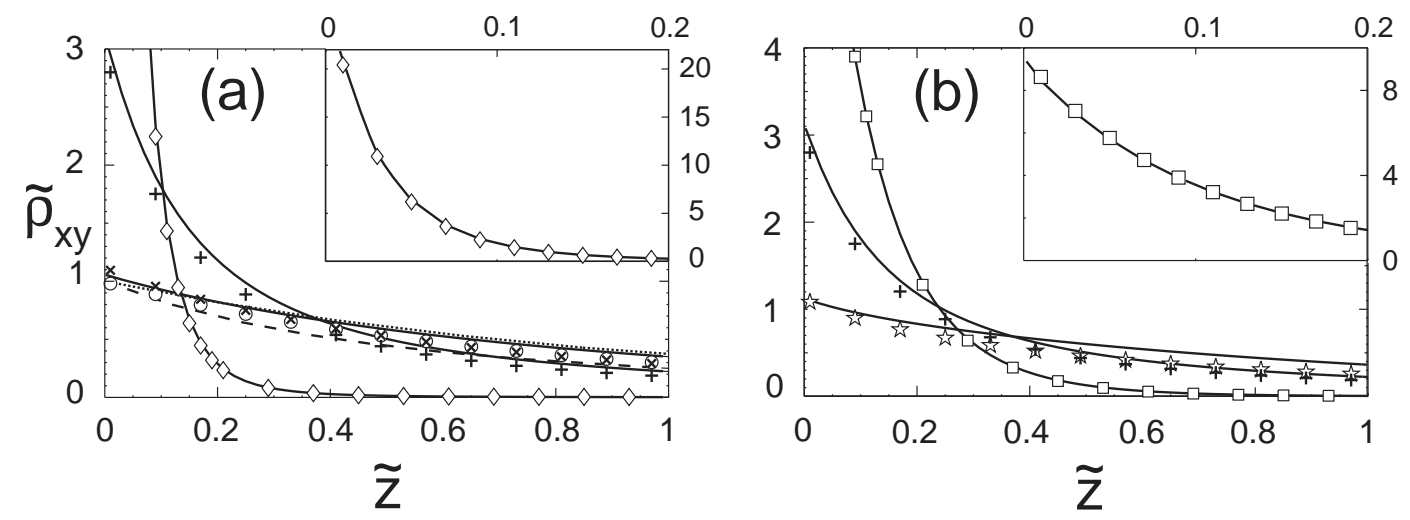

Fig. 2. - Counterion density profile $\widetilde{\rho}_{x y}$ (averaged in the $x y$-plane) as a function of $\widetilde{z} \equiv z / \mu$ for $q=Q$ and without dielectric jump $(\Delta=0)$. a) MC results (symbols) and SC predictions (full lines) for $\Xi=10$ and $D / a=0.06$ (diamonds and inset), $D / a=0.12$ (plus symbols), $D / a=0.24$ (crosses). The circles, dashed and dotted lines denote, respectively, the MC data, PB and SC predictions for $D / a=\infty$. b) MC results (symbols) and SC predictions (full lines) for $D / a=0.12$ and $\Xi=1$ (stars), $\Xi=10$ (plus symbols) and $\Xi=100$ (squares and inset). Error bars are smaller than symbol size.

density distribution is given by[18]

$$
\widetilde{\rho}(\widetilde{\mathbf{r}})=\frac{\rho(\widetilde{\mathbf{r}})}{2 \pi \ell_{B} \sigma_{s}^{2}}=\Lambda \mathrm{e}^{-\widetilde{u}(\widetilde{\mathbf{r}})}+\mathcal{O}\left(\Xi^{-1}\right)
$$

The factor $\Lambda$ is the fugacity of counterions, determined by the normalization condition $\int \mathrm{d} \widetilde{\mathbf{r}} \widetilde{\rho}(\widetilde{\mathbf{r}}) / \widetilde{L}^{2}=1$, where $\widetilde{L}$ is the lateral system size, equivalent to the condition of global electroneutrality[18]. The function $\widetilde{u}$, given by

$$
\widetilde{u}(\widetilde{\mathbf{r}})=-\Xi(1+\Delta) \frac{Q}{q} \sum_{\alpha} \frac{1}{\left|\widetilde{\mathbf{r}}-\widetilde{\mathbf{R}}_{\alpha}\right|}+\frac{\Xi \Delta}{4 \widetilde{z}}
$$

is the electrostatic potential created at position $\widetilde{\mathbf{r}}$ by the surface ions at lattice positions $\widetilde{\mathbf{R}}_{\alpha}$ (with $\widetilde{Z}_{\alpha}=-\widetilde{D}$ ) plus the counterion-image interaction. In previous studies we demonstrated that the SC approach becomes quantitatively accurate for coupling strengths larger than $\Xi \approx 100[16]$. The discreteness of surface charges tends to decouple different counterions from each other, as witnessed by the snapshots shown in Fig.1b. One would therefore expect corrections to the leading term of the systematic expansion in Eq.(2), which come from correlations between counterions, to be weakened and the SC approach to perform even better in the presence of modulated surface charges, as indeed borne out by our data. In the limit $D / a \rightarrow \infty$, the smeared-out case, Eq. (3) reduces to $\widetilde{u}(\widetilde{\mathbf{r}})=(1+\Delta) \widetilde{z}+\Xi \Delta /(4 \widetilde{z})$.

We first discuss our results in the absence of a dielectric jump $(\Delta=0)$ and identical valences of surface and counterions $(Q=q)$. In Fig. 2a we fix $\Xi=10$ while $D / a$ is varied; in Fig. $2 \mathrm{~b}$ we fix $D / a=0.12$ while $\Xi$ is varied. Clearly, the counterion density profiles $\widetilde{\rho}_{x y}(\widetilde{z}$ ) (which are averaged in the $x y$-plane) are very sensitive to both the coupling constant $\Xi$ and the ratio $D / a$. For $\Xi=10$ and $D / a=0.24$ (crosses in Fig. 2a) the discretization has a small effect, as the data almost coincide with the MC results for the smeared-out case $D / a \rightarrow \infty$ (circles). With the rescaling of Fig.2a, the difference between the smeared-out SC and PB profiles (dotted and broken lines, respectively) is in fact rather small compared to the effects of surface-charge modulation, and the smeared-out data (circles) are somewhat in between the $\mathrm{SC}$ and $\mathrm{PB}$ predictions, demonstrating that $\Xi=10$ is in the crossover regime between 


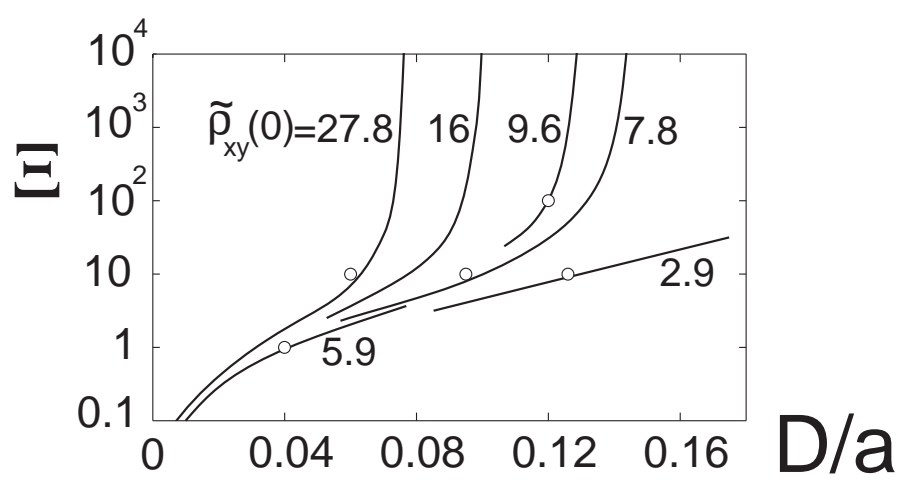

Fig. 3. - Contour plots of constant contact counterion density $\widetilde{\rho}_{x y}(\widetilde{z}=0)$ as a function of the coupling constant $\Xi$ and the ratio $D / a$ for $\Delta=0$ and $Q=q$. The lines follow from the SC theory via Eq. (2), while the open circles are obtained from MC data via extrapolation leading to $\widetilde{\rho}_{x y}(0)=2.9,5.9,7.8$, 9.6 and 27.8. For $D / a \rightarrow \infty$ as well as $\Xi \rightarrow 0$ the smeared-out assumption for the surface charge distribution, characterized by $\widetilde{\rho}_{x y}(0)=1$, is valid.

strong and weak coupling[16]. For $D / a=\infty$ and $\Delta=0$ the contact-value theorem predicts a rescaled contact density of unity, $\widetilde{\rho}_{x y}(\widetilde{z}=0)=1$, as confirmed by simulations at various values of $\Xi[16]$. However, as $D / a$ becomes smaller there is a greater accumulation of counterions in the immediate vicinity of the charged surface, and the laterally averaged contact density can be several times larger than unity. This is reflected by a high lateral correlation between counterions and the surface charges, see Fig. $1 \mathrm{~b}$. As $D / a$ becomes smaller, the SC predictions as defined in Eqs. (2) and (3) (solid lines in Fig. 2a) show progressively better agreement with the MC results. Similarly, in Fig. $2 \mathrm{~b}$ for fixed $D / a=0.12$, the contact density rises while the agreement between MC data and SC predictions becomes quantitative as $\Xi$ is increased.

These results are summarized in Fig. 3, where contour plots of constant contact density $\widetilde{\rho}_{x y}(\widetilde{z}=0)$ are shown as a function of $\Xi$ and $D / a$. The lines were obtained from the SC theory via Eq. (2) and agree well with the MC results (open circles), obtained from density profiles via extrapolation. In the limit $D / a \rightarrow 0$ and finite coupling $\Xi$ the counterions collapse onto the surface charges and the density at contact diverges, while for fixed $D / a$ and in the limit $\Xi \rightarrow \infty$ the contact density saturates at a finite value. As $D / a$ grows (or $\Xi$ becomes smaller), the smeared-out assumption becomes valid and $\widetilde{\rho}_{x y}(0)$ approaches unity.

We finally lift the restriction to symmetric surface/counterion valences $(q=Q)$ and to vanishing dielectric jump $(\Delta=0)$. In Fig. 4a we show density profiles for $\Xi Q^{3} / q^{3}=10$, $D / a=0.12, \Delta=0$ with varying valence ratios of $q / Q=1$ (plus symbols, already featured in Fig.2), $q / Q=2$ (stars), $q / Q=4$ (triangles) and $q / Q=16$ (squares). The inhomogeneity of the surface charge distribution becomes more important as the counterion valence increases. The SC results (solid lines) capture this trend and quantitatively agree with simulation data. The contact density $\widetilde{\rho}_{x y}(0)$ saturates at a constant value as $q / Q \rightarrow \infty$ (see inset). For the case where a dielectric jump is present, we choose $\varepsilon_{>}=80$ and $\varepsilon_{<}=2$, leading to $\Delta=0.95$ and corresponding to a charged hydrocarbon substrate in contact with water. Since $\Delta$ is positive, the interaction between the counterions and their images is repulsive and competes with the attractive interaction due to the surface charges, see Eq. (1). Consequently, the single-ion potential $\widetilde{u}(\widetilde{\mathbf{r}})$, Eq.(3), exhibits a minimum at a finite distance from the wall, and one expects the maximum in the density profile to be displaced from the wall, in agreement with previous results[20, 21] (however, one should note that the resulting density profile depends strongly on details of the geometry and position of the dielectric boundary). Fig. 4b shows 

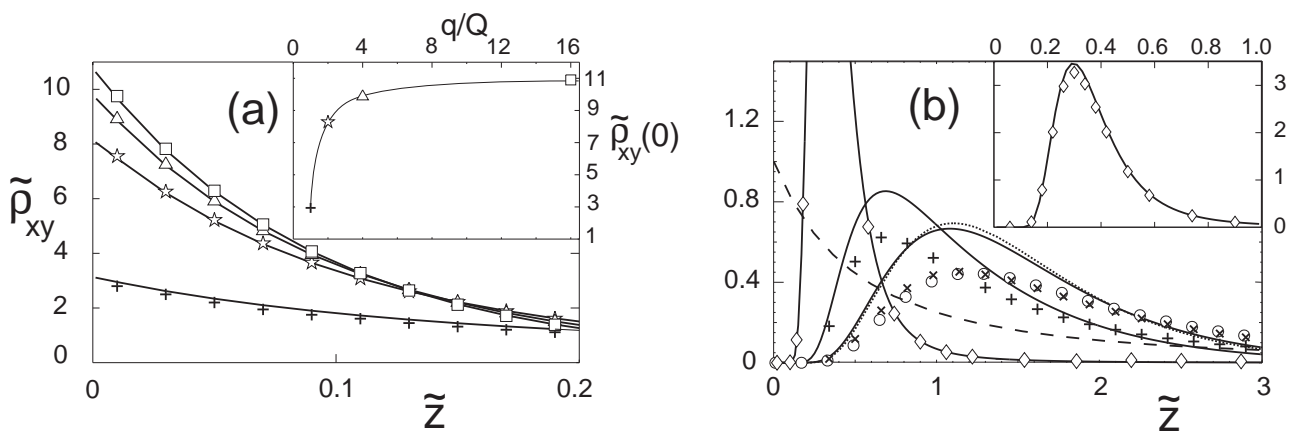

Fig. 4. - a) Counterion density profiles $\widetilde{\rho}_{x y}(\widetilde{z})$ for $\Xi Q^{3} / q^{3}=10, D / a=0.12, \Delta=0$ and different values of the valence ratio $q / Q$. Full lines correspond to the $\mathrm{SC}$ predictions and symbols to MC results for $q / Q=1$ (plus symbols), $q / Q=2$ (stars), $q / Q=4$ (triangles) and $q / Q=16$ (squares). b) $\mathrm{MC}$ results (symbols) and SC predictions (full lines) for the counterion density profile for $\Xi=10, q=Q$ in the presence of a dielectric jump, $\Delta=0.95$, for $D / a=0.06$ (diamonds and inset), $D / a=0.12$ (plus symbols) and $D / a=0.24$ (crosses). The circles, dashed and dotted lines denote, respectively, $\mathrm{MC}$ data, $\mathrm{PB}$ and $\mathrm{SC}$ predictions in the smeared-out limit, $D / a \rightarrow \infty$.

the counterion density profile for $\Xi=10, q=Q, \Delta=0.95$ and the same values of $D / a$ as in Fig. 2a, namely $D / a=0.06,0.12$ and 0.24 . The corresponding SC predictions (shown as solid lines) locate correctly the position of the maximum in the density, though the agreement is not as good as without the dielectric jump for the larger values of $D / a$. The data for the smeared-out case, $D / a=\infty$ (open circles) exhibit a maximum close to $\widetilde{z}=\sqrt{\Xi \Delta /(4(1+\Delta))}$, the $\mathrm{SC}$ prediction, although the SC profile for this case (dotted line) overestimates the amount of counterions close to the wall. Obviously, the SC theory does not take into account the repulsion felt by a counterion due to the presence of the images of other counterions, which explains why SC theory performs at the same value of $\Xi$ better in the absence of a dielectric jump (compare Fig.2). Higher order corrections to the asymptotic SC theory would therefore be needed to quantitatively match the data. As is well-known, PB theory (shown as a broken line) is insensitive to the presence of a dielectric jump in the smeared-out limit $D / a \rightarrow \infty$ and completely misses the shape of the density profile[20, 21].

To conclude, we have studied both analytically and numerically the distribution of counterions close to an inhomogeneously charged surface. The charge inhomogeneity affects the counterion distribution strongly for pronounced charge modulation as well as when the coupling constant $\Xi$ is large. As summarized in Fig. 3 in the absence of a dielectric discontinuity $(\Delta=0)$, the laterally averaged counterion contact density is much larger than the smeared-out value at decreasing $D / a$ (equivalent to a strongly charge-modulated substrate) and at increasing coupling constant $\Xi$, and counterions become strongly correlated with the surface charges. The same effect is reflected in the counterion density profiles, which under such conditions decay faster to zero as one moves away from the substrate, see Fig.2.

This has direct experimental consequences: Very recently the counterion density profile (measured using ellipsometry) at a surfactant monolayer with a high surface charge density (determined via second-harmonic generation) could be described by PB profiles only by assuming a fraction of counterions to be bound to the monolayer[19], similar to what we find. Our results suggest to reconsider the traditional picture of a Stern layer, where the counterion surface concentration is subject to electrostatic and specific surface attraction and packing constraints, but where the influence of lateral surface charge modulation (which is always present experimentally and leads to sizable effects as shown here) is typically neglected. One should emphasize the experimental relevance of our parameters: for a system with $\sigma_{s} \simeq 1 / 257 \AA^{-2}$ 
and divalent counterions in water (which at room temperature leads to $\Xi=10$ ), the surface ions are on average at a distance $a \simeq 16 \AA$ from each other. For a minimal distance between surface ions and counterions of $D \simeq 2 \AA$ (noting that at close contact the hydrated water shell is stripped off) the ratio $D / a=0.12$ is obtained; as our results demonstrate, the inhomogeneous character of the surface charges leads to pronounced deviations from the smeared-out case.

Other relevant factors, which we will consider in future work, include the possibility of the counterions to actually penetrate between the surface ions, different surface charge patterns (annealed and various quenched geometries), and additional interactions between surface groups and counterions (like van der Waals or solvation forces) and between counterions themselves (most importantly hard core interactions, which to leading order within the SC approach simply shift the density profile away from the surface). The advantage of the currently employed model, though being quite crude and neglecting a number of important effects, is that its simplicity allows for a global analysis, encompassing different and limiting values of the coupling strength and the degree of substrate charge modulation. In a complementary study, similar numerical calculations have been recently analyzed within a modified PB approach[26].

We thank H. Motschmann and R. Teppner for useful discussions. AGM acknowledges financial support from the DFG Schwerpunkt Polyelektrolyte.

\section{REFERENCES}

[1] H. Kihira, N. Ryde, and E. Matijević, J. Chem. Soc. Faraday Trans. 88, 2379 (1992).

[2] G. M. Litton and T. M. Olson, J. Colloid Interface Sci. 165, 522 (1994).

[3] J. Y. Walz, Adv. Colloid Interface Sci. 74, 119 (1998).

[4] J. Aizenberg, P. V. Braun, and P. Wiltzius, Phys. Rev. Lett. 84, 2997 (2000).

[5] H. Clausen-Schaumann and H.E. Gaub, Langmuir 15, 8246 (1999).

[6] P. Richmond, J. Chem. Soc. Faraday Trans. II 71, 1154 (1975).

[7] D. Y. Chan, D. J. Mitchell, and B. W. Ninham, J. Chem. Phys. 72, 5159 (1980).

[8] M. Kostoglou and A. J. Karabelas, J. Colloid Interface Sci. 151, 534 (1992).

[9] R. M. Peitzsch, M. Eisenberg, K. A. Sharp, and S. McLaughlin, Biophys. J. 68, 729 (1995).

[10] T.T. Nguyen, A.Y. Grosberg, B.I. Shklovskii, J. Chem. Phys., 113 (2000) 1110.

[11] R. Kjellander and S. Marčelja, J. Chem. Phys. 88, 7138 (1988).

[12] O. Gonzalez-Amezcua, M. Hernandez-Contreras, P. Pincus, Phys. Rev. E 64, 041603 (2001).

[13] W. van Megen and I. Snook, J. Chem. Phys. 73, 4656 (1980).

[14] T. Åkesson and B. Jönsson, J. Phys. Chem. 89, 2401 (1985); H. Wennerström and B. Jönsson, J. Phys. France 49, 1033 (1988).

[15] R. Messina, C. Holm, and K. Kremer, Eur. Phys. J. E 4, 363 (2001).

[16] A. G. Moreira and R. R. Netz, Europhys. Lett. 52, 705 (2000).

[17] A. G. Moreira and R. R. Netz, Phys. Rev. Lett. 87, 078301 (2001).

[18] R. R. Netz, Eur. Phys. J. E 5, 557 (2001).

[19] R. Teppner, K. Haage, D. Wantke, and H. Motschmann, J. Phys. Chem. B 104, 11489 (2000).

[20] R. Kjellander and S. Marčelja, Chem. Phys. Lett. 112, 49 (1984).

[21] D. Bratko, B. Jönsson, and H. Wennerström, Chem. Phys. Lett. 128, 449 (1986).

[22] P. Attard, D. J. Mitchell, and B. W. Ninham, J. Chem. Phys. 88, 4987 (1988).

[23] R. Podgornik and B. Žekš, J. Chem. Soc., Faraday Trans. 2 84, 611 (1988).

[24] J. D. Jackson, Classical Electromagnetism, 2nd ed. (John Wiley and Sons, New York, 1975).

[25] J. Lekner, Phys. A 176, 485 (1991); R. Sperb, Mol. Simul. 20, 179 (1998).

[26] D.B. Lukatsky, S.A. Safran, and P. Pincus, to be published. 